\title{
MECHANICAL ASPECTS OF THE CHARPY IMPACT TEST
}

A. Rossoll*, C. Berdin*, P. Forget $\dagger$, C. Prioul*, and B. Marini $\dagger$

\begin{abstract}
A comparative experimental and FEM study has been performed, in order to investigate dynamic and constraint aspects of the Charpy test. Testing of standard V-notch Charpy specimens was performed under dynamic and static loading conditions. 2-D plane strain and 3-D models were used in numerical analysis. In order to incorporate strain-rate effects, an elastic-viscoplastic constitutive equation has been applied, based on actual test data obtained for a low-alloy structural steel. Fully dynamic analysis clearly indicated inertial effects. Modal analysis enabled the identification of the origin of the oscillations on the load-displacement curve as beam vibration of the specimen resulting from interaction with the elastic striker.
\end{abstract}

\section{INTRODUCTION}

The instrumented Charpy impact test is largely applied in industry, due to the fact that it is easy to conduct and results can be obtained quickly and with little cost. Yet it seems that among some users there is still some uncertainty about the origin of the oscillations occurring on the load vs. displacement records. Furthermore, some doubt exists concerning the actual state of constraint in this rather small type of specimen. The study of inertial effects is of some interest in the cleavage fracture domain, since failure will occur after a relatively short loading time, thus possibly not allowing for oscillations to dampen out quickly enough. This study is performed, in parallel with a comparative study by Tahar, Piques and Forget (1), as a first step in the framework of a common research programme devoted to modelling of the Charpy test based on a "local" criterion for cleavage fracture.

\section{EXPERIMENTAL PROCEDURE}

The material used for the investigation was taken from a nozzle cut-out of a nuclear pressure vessel made of A $508 \mathrm{Cl} .3 \mathrm{steel}$. This is a $0.16 \mathrm{C} 1.3 \mathrm{Mn} 0.7 \mathrm{Ni} 0.5 \mathrm{Mo}$

* Ecole Centrale de Paris, Laboratoire MSS-MAT, CNRS U.R.A. n 850, F-92295 Châtenay-Malabry Cedex, France.

† CEA Saclay, CEREM, SRMA, F-91 191 Gif-sur-Yvette Cedex, France. 
structural steel used for heavy forged components in nuclear industry. Heat treatment includes 2 austenitisations followed by water quenching and tempering, and final stress relief.

\section{Parameter Identification of the Constitutive Equations}

Static tensile tests $\left(\dot{\varepsilon}=4 \cdot 10^{-4} \mathrm{~s}^{-1}\right)$ have been conducted at temperatures ranging from $-196{ }^{\circ} \mathrm{C}$ up to room temperature. A Liiders strain of about $1 \%$ was found. Since the development of Lüders bands is considered to be a characteristic of tensile testing conditions, and not an intrinsic characteristic of the material per se, an extrapolation of the strain-hardening part of the stress-strain curve to zero plastic strain was performed, in order to receive the "true" material response. Thus a new yield limit was defined (see figure 1). This procedure, which is based on dislocation movement considerations, has already been documented in literature, for instance, amongst others, by Lindley (2).

Since the material just below the notch root of a Charpy specimen exhibits high strain rate during deformation, it was necessary to investigate the strain-rate sensitivity of the material. Compressive testing at an approximate strain rate of $1000 s^{-1}$ on a Hopkinson bar device. Additionally, quasistatic $\left(4.10^{-3} \mathrm{~s}^{-1}\right)$ and intermediate strain rate $\left(1 \mathrm{~s}^{-1}\right)$ compressive tests were performed on an INSTRON hydraulic testing machine, at temperatures between $-150^{\circ} \mathrm{C}$ and an $100^{\circ} \mathrm{C}$. $+100^{\circ} \mathrm{C}$. Only the plaster virtually "isothermal" conditions. These tests allowed to numerically identify the parameters for the elastic-viscoplastic material law in additive formulation proposed by ABAQUS FEM software.

\section{Impact Testing}

Charpy tests on three different standard instrumented impact pendulum

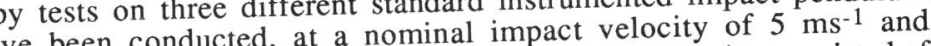
devices have been conducted, at a nominal impact velocity of temperatures ranging from $-90^{\circ} \mathrm{C}$ to room temperature. Instrumentation consisted of measuring striker displacement and force. Additionally, a few tests on specime of equipped with strain gauges have been undertaken, in o
the apparent oscillations on the load-displacement record.

\section{Static Testing}

Static testing of standard Charpy specimens was performed on an INSTRON hydraulic testing machine. Both striker and supports had been machined according hydras imposed. to Charpy testing standards. A crosshead spe to measure the notch opening, and Instrumentation also included a clip gauge to measure the notch opening, and $-30^{\circ} \mathrm{C}$ were studied.

\section{NUMERICAL ANALYSIS}

Mesh. 2-D and 3-D FE meshes have been generated by means of I-DEAS software In 2-D up to 2300 4-noded, and in 3-D 8700 8-noded linear isoparametric elements 作 with reduced integration have been used. Due to symmetry, only half (2-Dists of 10 
layers of elements in the thickness direction, with decreasing node distance towards the outer surface. A fine mesh is used for modelling contact surfaces and notch section of the specimen. Rigid surface contact elements have been used to model striker and supports. A coefficient of friction of 0,1 was assumed. Loading of the specimen is modelled by imposing a fixed displacement (for static testing simulation) or velocity (for dynamic testing simulation) to the striker. In fully dynamic analysis, impact velocity is imposed upon the rigid striker contact surface via a spring element, thus simulating machine compliance. A mixed 2-D/3-D element approach using constraint equations for the 3-D part had to be given up, since it was found that the 3-D part would need to be very large in order to yield a correct solution.

Analysis. The ABAQUS 5.4 finite element analysis program with implicit integration scheme has been used. Finite strains have been accounted for, thus allowing the description of local stress and strain fields in the heavily deformed notch region of the Charpy specimen. Calculations of impacted specimens included the following features : $5 \mathrm{~ms}^{-1}$ striker velocity imposed ; elastic-viscoplastic material behaviour ; quasistatic or fully dynamic (including inertia terms ; 2-D only) solution procedure. Fully adiabatic conditions have been considered, thus not allowing any transfer of the heat generated by plastic deformation, since the characteristic heat diffusion time is considered to be about one order of magnitude longer than the duration of an impact test. Any numerical simulation has been terminated after a displacement of the striker of $2 \mathrm{~mm}$, since testing showed that, in the temperature regime under investigation, failure of the specimen occurs within this range.

\section{RESULTS AND DISCUSSION}

In order to validate the numerical models, experimental and numerical global response results (load-displacement) are compared in figures 2 and 3 . In the simulation of static testing, a "stick-slip" effect appears, which seems an artefact due to the contact algorithm, since it does not appear on experimental curves, but does so on results of calculation even assuming no friction. No ductile damage has been taken into account, which is supposed to develop after a deflection in the order of $0.5 \mathrm{~mm}$, according to the observation of the potential drop signal. Consequently, a slight overestimation of the numerically obtained global response curve for larger displacements arises. In conclusion, the 3-D modelling seems rather reliable. The global dynamic response of the specimen largely overestimates the reaction force. This is due to the 2-D plane strain hypothesis applied for dynamic calculations, since 3-D calculation in fully dynamic conditions would be extremely costly.

Modal analysis enabled the identification of the origin of the oscillations as beam vibration of the specimen resulting from interaction with the "elastic" striker, phenomenon which has already been specified through different mass-spring analyses and beam solutions, e.g. by Sahraoui and Gillaizeau (3). It was found that amplitude and frequency of the apparent oscillations on the load-displacement record are greatly influenced by the stiffness of the experimental device (i.e. machine compliance).

The evolution of the longitudinal strain rate in the first element close to the notch root is plotted in figure 4 . It shows strong fluctuations for the dynamic analysis, but influence of these fluctuations on strain hardening is considered to be 
eneral level. The apparent strain rate is lower for

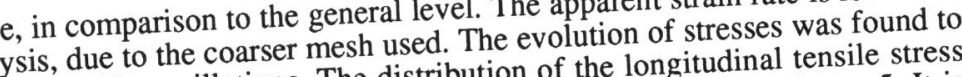
the distribution of the lonitudinal tensile stress exhibit negligeable oscillations. The of the specimen is plotted in figure 5. It is along the ligament in the centre platic (elastic-plastic compared for deflections of 0.5 and 2 (elastic-viscoplastic material behaviour) testing material behaviour) and dynamic (elastic-viscople much higher, due to strain rate conditions. Stresses obtained in dynamic testing aridered temperature of $-90^{\circ} \mathrm{C}$, the hardening. In figure 6 it is shown that, for a conalysis closely follows the solution longitudinal tensile stress in plane strain $2-\mathrm{D}$.

In figure 7 the temperature distribution in the notch root region due to adiabatic heating in dynamic testing can be seen, for a deflection of $0.5 \mathrm{~mm}$, and an initial temperature of $-90^{\circ} \mathrm{C}$ (2-D plane strain analysis). It can be stated that the temperature elevation is already quite important, but confined to a very small zon at Figure 8 shows an energy balance analysis of a dynatic energy is rather low, in $-90^{\circ} \mathrm{C}$ : It can be seen that, after two oscillations, kinetic energy is rathe time taken comparison to total energy; this means that, if the specimen fial, and the energy obtained by for two oscillations, the energy measured on the dial, and consumed by deformation integrating the force-displacement signal is nearly entirely consumed by defor. and fracture of the specimen; so a static evaluation procedure can be applied. The "classical" $3 \tau$ criterion for validity of Charpy impact tests (e.g. be considered too requires that fracture does not occur before 3 oscillations. It could be consilu level before failure of conservative,

the specimen.

\section{CONCLUSIONS} It has been shown that numerical modelling of the Charpy impact tests yields quite reasonable results. Fully dynamic ansement records. Inertial fluctuations of stresses experimentally obtained load-displacement records. Iicty. Local stresses in 2-D plane in the notch region dampen out quickly due to plasticity. Llane of the specimen in 3-D strain analysis were found to follow those in the center of $-90^{\circ} \mathrm{C}$ considered, a 2-D analysis. It can be concluded that, for a temperature employed, in order to apply a plane strain quasistatic evaluation

local cleavage fracture criterion.

\section{ACKNOWLEDGEMENTS}

The authors are grateful to EdF Les Renardières for providing the specimens, and to ETCA Arcueil for conducting the compressive tests. Technical support of Mr. Karl Fatrdla is gratefully acknowledged.

\section{REFERENCES}

Tahar, M., Piques, R. and Forget, P., this conference.

Lindley, T.C. and Smallman, R.E., Acta Met., Vol. 11, May 1963, pp. 361-

(3) Sahraoui, S. and Gillaizeau, F., Engng Fracture Mech., Vol. 33, No. 6 , 1989, pp. 871-876.

W.L., JTEVA, Vol. 6, No. 1, Jan. 1978, pp. 29-34. 

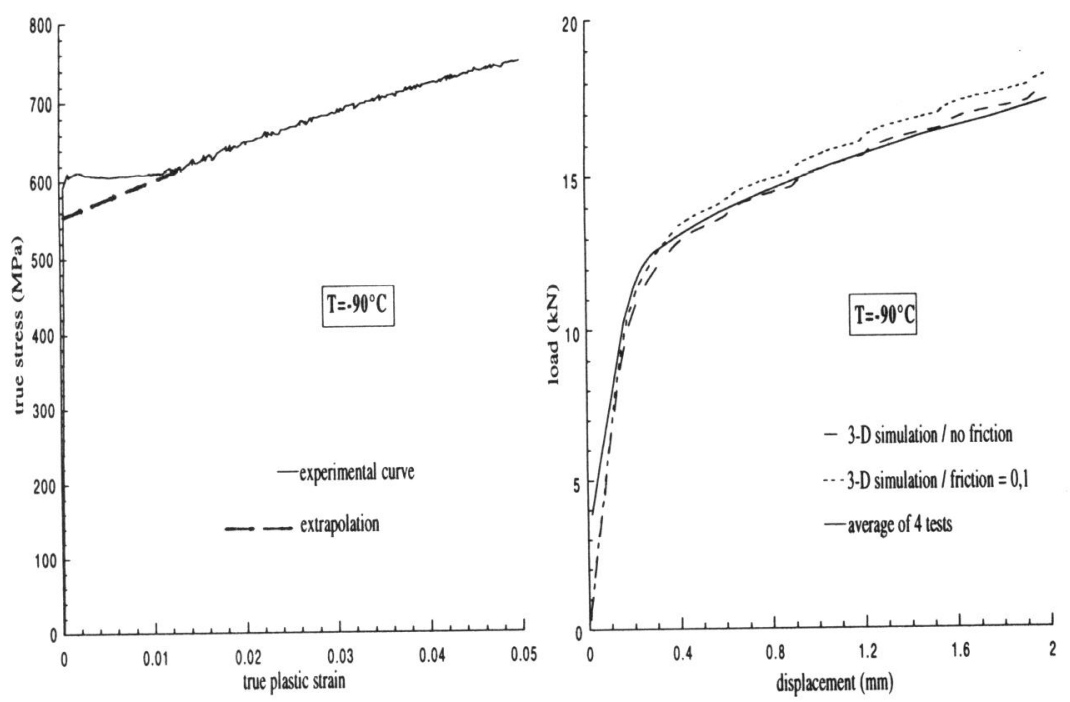

Figure 1 Extrapolation technique used for redefining the yield limit Figure 2 "Static" experimental and
numerical global response

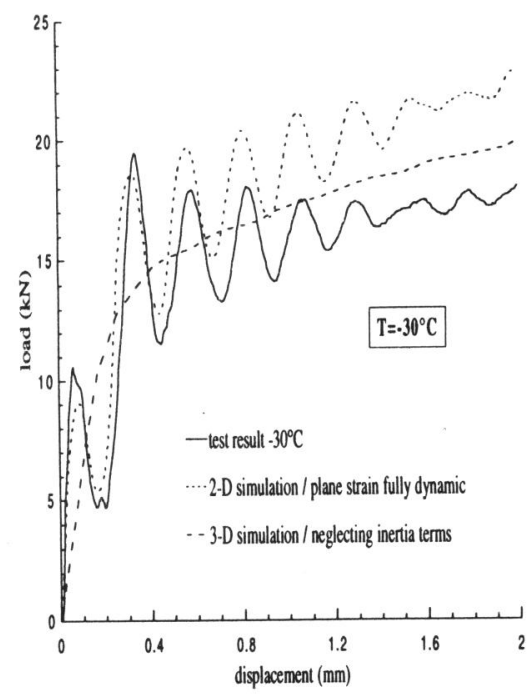

rigure 3 "Dynamic experimental and numerical global response

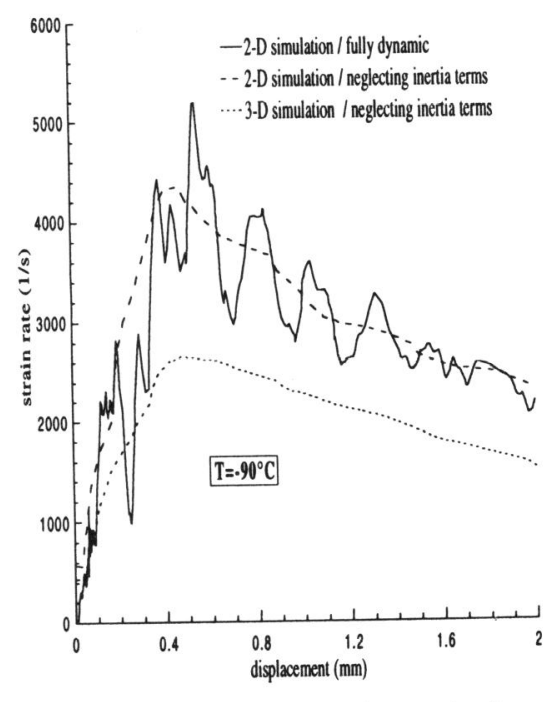

Figure 4 Longitudinal strain rate in the notch root element 


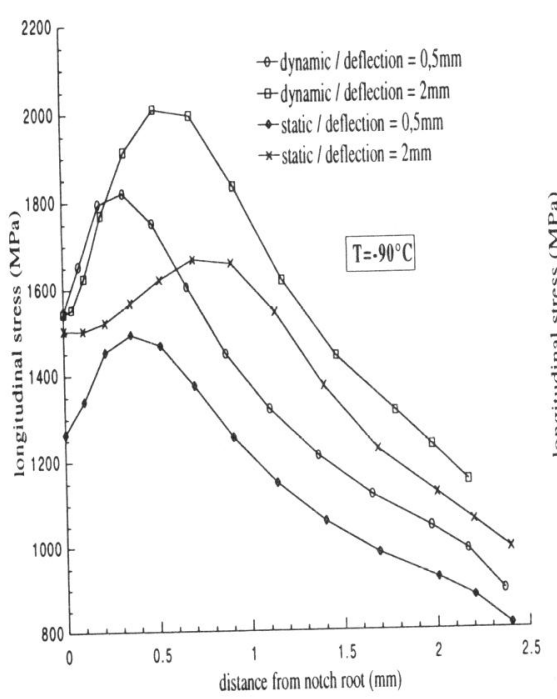

Figure 5 Longitudinal tensile stress : 3-D analysis / center plane

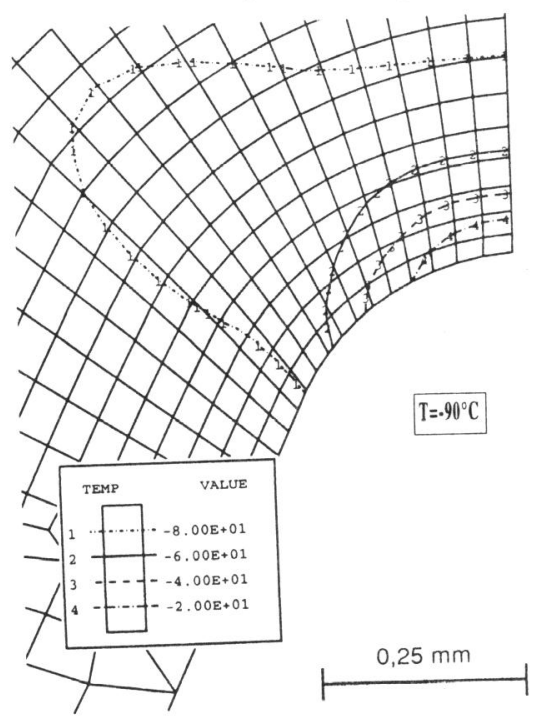

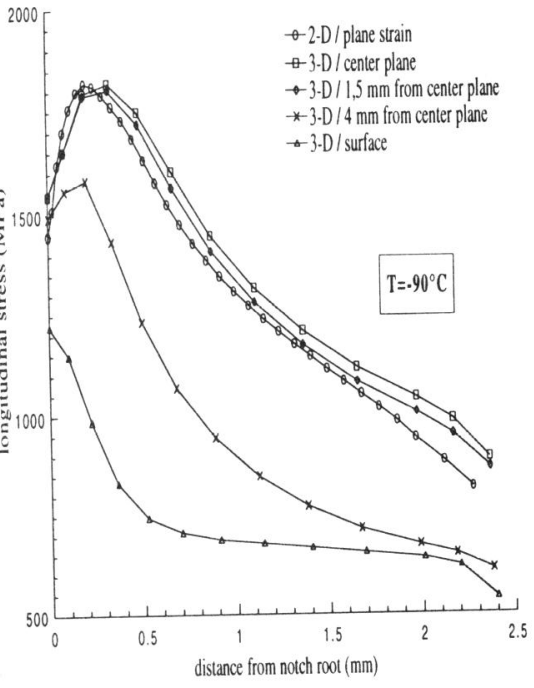

Figure 6 Longitudinal tensile stress : different plane sections and 2-D

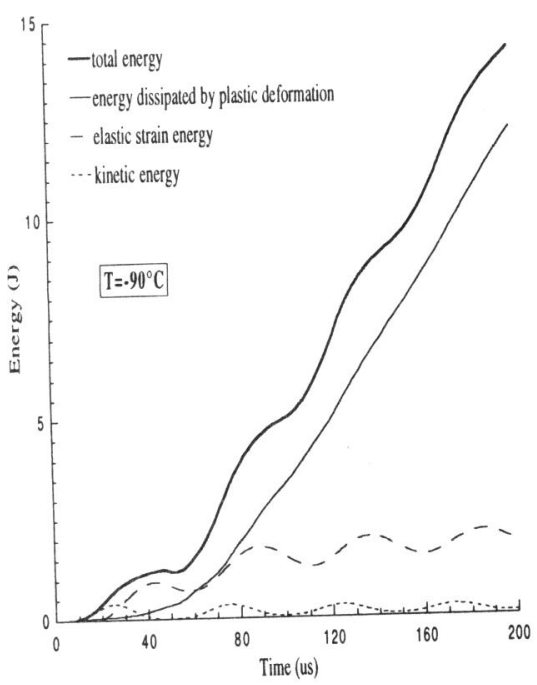

Figure 7 Temperature ahead of the notch Figure 8 Energy balance for 2-D fully after a deflection of $0.5 \mathrm{~mm}$ dynamic analysis 